\title{
Community Case Managers' (CCMs) Perspectives' Collaborating With Primary Care Services When Managing Complex Patients in the Community: A Qualitative Study.
}

Gilbert Yeo ( $\sim$ Gilbert_ts_yeo@nhgp.com.sg )

National Healthcare Group Polyclinics

Predeebha Kannan

National Healthcare Group Polyclinics

Eng Sing Lee

National Healthcare Group Polyclinics

Helen Smith

Nanyang Technological University

\section{Research Article}

Keywords: community case managers (CCMs), collaboration, collaborative care, primary care services, community care, challenges, complex patients, Singapore

Posted Date: January 22nd, 2021

DOI: https://doi.org/10.21203/rs.3.rs-146440/v1

License: (c) (1) This work is licensed under a Creative Commons Attribution 4.0 International License.

Read Full License 


\section{Abstract}

Background: Strong collaboration between community case managers (CCMs) and medical professionals are vital for the continuity of complex patients' care in the community. Key challenges can influence or even deter successful collaboration.

Objective: The objective of this qualitative study was to understand the challenges encountered by CCMs when collaborating with primary care services.

Methods: This exploratory qualitative descriptive study used individual in-depth interviews. CCMs were selected using maximum variation and snowball sampling. The interviews were semi-structured, guided by a topic guide. Data were analyzed alongside ongoing data collection. Thematic content analysis was used to analyze the transcripts. Initial codes were obtained independently. These codes were then further explored, clarified and iteratively grouped into themes.

Results: Fourteen individual in-depth interviews were conducted. Six themes emerged from the data i.e. self-identity, patient factor, inter-professional factor, collaborative culture, confidentiality and organizational structure. Challenges that resonated with previous studies were self-identity, interprofessional factors and confidentiality, whereas other challenges such as patient factors, collaborative culture, and organizational structure, were unique to Singapore's health care landscape.

Conclusions: Significant challenges were encountered by CCMs when collaborating with primary care services. Understanding these challenges is key to refining intervention in current models of comprehensive community care between medical and non-medical professionals.

\section{Introduction}

Primary care often acts as the hub for coordinating care of patients with multiple chronic conditions and also those confronted with complex psychosocial problems intertwined with medical problems. Early definitions of complex patients focused on factors such as the number of chronic diseases or medications.[1] More recent definitions of complex patients incorporate mental health, social influences, and economic factors that substantially affect chronic disease outcomes.[2, 3] The Agency for Integrated Care (AIC) of Singapore defines complex patients as those fulfilling at least two of three domains; complex medical issues ( $\geq$ three chronic conditions or advanced disease), functional impairment (requiring assistance in $\geq$ one activities of daily living or cognitive impairment) or psychosocial impairment (caregiver, family, financial, social isolation or psychological issues).[4]

In Singapore, the primary care services are provided by 20 polyclinics belonging to three different healthcare clusters with around 400 doctors and about 1,700 General Practitioner (GP) clinics for a population of 5.8 million. The proportion of attendances attributed to chronic disease has increased from $18 \%$ in 2010 to $27 \%$ in 2014 ; with care for elderly patients increasing from $10 \%$ in 2010 to $15 \%$ in 2014.[5] Though the primary care services are nestled in the community, they are still confined within the 
environment of a consultation room. Primary healthcare providers do not usually have the resources to follow up on complex patients in their place of residence in the community. This however, could be achieved by tapping on the resources and expertise of social work services through case management.

The Case Management Society of Singapore (CMSS) defines case management as a collaborative process that utilizes a comprehensive and holistic assessment that aims to achieve optimal care in an appropriate care setting with cost-effective outcomes.[6-8] The terms social workers and community case managers (CCMs) have been used interchangeably, depending on the place of practice;[9] indeed, case management is an integral part of social workers role.[10] Integrating CCMs into the primary care setting offers an approach to address shortfalls of patient care in the community. There are ample empirical studies supporting the key roles and benefits of case management [11-15] but studies understanding the challenges of inter-professional collaboration between medical professions and social workers are lacking in the medical literature. This may suggest the existence of professional apartheid between primary healthcare workers and CCMs despite the widely accepted benefits of collaborative care of complex patients.

From a theoretical lens, this study draws on the concept of social identity theory (SIT) expounded by Tajfel \& Turner.[16] This widely accepted theory has contributed to the understanding of identity and intergroup relationships. According to SIT, identity is formed through the three processes of social categorization, social identification and social comparison.

Abramson \& Mizrahi [17] developed a typology of collaborators from a qualitative study that addressed professional behavior between social workers and physicians to understand the complexities of interdisciplinary relationships. Understanding the perspectives of social workers and physicians can be summarized into three continuum models of collaboration namely traditional, transitional and transformational, each with its own challenges and strengths. Unfortunately, the study findings have limited applicability in primary care since the study was conducted in acute hospitals. A systematic review by Gabrielova \& Veleminsky [18], also involving hospitals, revealed four main challenges experienced by social workers; namely theoretical differences, various professional perspectives, lack of knowledge and weak communication. Keefe, B. et al., identified two themes concerning community social workers [19] with focus group discussions involving primary care physicians and nurses. The first theme was the perceived role of the social worker with care coordination, placement, and community-based services as sub-themes. The second theme reported the challenges of having a social worker in the primary care team, including additional time burden and pressures on office space. However, this study was confined to doctors and nurses and did not include the CCMs' perspectives. Another paper regarding the challenges of primary care case manager-physician collaboration by Netting \& Williams [14] included perspectives from both sides. The authors described three themes; namely relationships, differing role perspectives, and professional identity.

Currently in Singapore, CCMs are not involved with the care planning of complex patients with the primary care team and are often considered as non-members of the team. For many years, the social 
support for health services, including CCMs, were under the purview of the Ministry of Social and Family Development. In recognizing the importance of integrating health and social services, the Ministry of Health has taken oversight of both services since April 2018. [20] There is now the potential to engage and integrate CCMs into the primary care team. This study aimed to understand the challenges encountered by CCMs when collaborating with primary care services.

\section{Methods}

\section{Participant sampling and ethics}

Individual in-depth interviews were conducted until the point of saturation where no new information emerged. CCMs were selected using maximum variation and snowball sampling. The inclusion criteria were (i) CCMs involved in managing complex patients in the community i.e., community organizations, voluntary welfare organizations or nursing homes; (ii) at least one year of experience working as a CCM and (iii) prior training in case management. CCMs were invited through invitation e-mails followed by telephone calls to confirm their willingness to participate. Their participation was voluntary.

\section{Data collection}

Ethics approval for this exploratory qualitative descriptive study was obtained from the National Healthcare Group Domain Specific Review Board (NHG DSRB) (Ref: 2018/00801) and methods were performed in accordance with the relevant guidelines and regulations laid by NHG DSRB. The study objectives and confidentiality of data were explained to all potential participants and a minimum of two days were provided for them to ask questions and consider participating in the study. All individual indepth interviews were conducted after the written informed consent forms were signed. The interviews were conducted in English in privacy at the CCMs' administration offices by a researcher (GTSY). Sociodemographic information and a brief anonymous self-introduction were obtained before commencing the interview. The individual in-depth interviews were semi-structured and guided by a topic guide. [21] The individual in-depth interviews were recorded digitally.

\section{Data analysis}

Each CCM was provided with a pseudonym. The audio-recorded interviews were transcribed verbatim. Thematic content analysis was used to analyze data. Data were analyzed alongside ongoing data collection. Initial codes were identified and developed independently by two researchers (GTSY \& PK) in an iterative process of reading and re-reading transcripts. These codes were then further explored, clarified and iteratively categorized into themes. Any disagreements were discussed between both researchers to reach a consensus. Conscious attempts of reflexivity were made by the researchers so as to be open to unexpected findings.

\section{Results}


Fourteen individual in-depth interviews were conducted. The length for each individual in-depth interview lasted about forty-five to sixty minutes. The mean age of the CCMs was 36 years (range: 24-50 years) and the majority of them were female $(n=12)$. The average number of years of practice as CCMs was five years (range: 2-12 years). As for the location of practice, four CCMs served island wide, eight served the central and northeast regions whereas one served the western and eastern regions respectively. They all had formal postgraduate training in social work, although they had diverse undergraduate training, for example in nursing, psychology and finance.

\section{Challenges faced by CCMs}

Six main themes and 16 subthemes were identified in the data (Table 1).

Table 1

Themes and sub-themes

\begin{tabular}{|ll|}
\hline Theme & Sub-themes \\
\hline Self-identity & $\begin{array}{l}\text { An undefined and multifaceted role } \\
\text { Under-recognized }\end{array}$ \\
\hline Intellectual disability \\
Physical disability \\
Financial obstacles
\end{tabular}

\section{Self-identity}

\section{An undefined and multifaceted role}


CCMs attributed many challenges arose because of the nature of their job, one which is multifaceted and undefined. They manage not only issues of patients but also family members, combined with many administrative tasks.

"Then in the office, we're doing the paperwork, a lot of like y'know, er, coordination, telephoning or via email that kind of thing, ya." (CCM 4)

"I think it's our role. Our role is quite [tsk], I think not very clearly defined, because we really help them with a range of things. Sometimes, even introducing them what we do is tough, y'know." (CCM 5)

"We er, most of the senior or their family member do not, maybe are not aware of the available resources in the community so we, we may sort of er, recommend, and introduce some of the available services." (CCM 7)

\section{Under-recognized}

CCMs were unanimous that the role of CCMs in the community is under-recognized.

"I think people don't recognize that we are the owners [pause] of the clients. They think that y'know, because we, we don't have the resource, or y'know, we're-we're like, just in the community..." (CCM 10)

“... because we are not being recognized as a community case manager. Singapore is not there yet. You talk about community care, shift care, whatever, but first of all, this title is it recognized? It's not!" (CCM 11)

\section{Patient Factors}

\section{Intellectual disability}

CCMs indicated that it was a challenge to manage the care of people with intellectual disabilities within primary care services.

"Ya, and I think a lot of doctors not really trained in ID (Intellectual Disability) sector. I mean, not only the polyclinic doctor but even some speci-, hospital doctor, they're not really trained in this sector so it's a lot of stumbling block for this sector." (CCM 4)

There was a lack of support and inadequate care for patients with intellectual disability and their caregivers.

"So we're worried how he has been coping in the community, especially with his medical con-, I mean autism... So, in the end we managed to get a MINDS (Movement for the Intellectually Disabled of Singapore) doctor to go in, ya, on a voluntary basis" (CCM 5)

\section{Physical disability}


Similarly, CCMs also described the challenges of helping patients with physical disability, as there were limited transport escort services to the clinic and limited resources for house calls.

"But, er... I think one... thing that we always overlook is that sometimes client, they don't... they're not able to get primary care because they're not able to get there physically, ya. Transport escort is always an issue, and we do not have the manpower..." (CCM 9)

"... of course they (doctors) cannot do home call. I mean, as in, do visit and all - so I... we have to try our best to bring down the client, that is the challenge. Sometimes client don't want to go down. Or client, just um, the functioning impairment is very difficult to bring down." (CCM 11)

\section{Financial obstacles}

CCMs attributed the higher cost of care for physically disabled patients, non-residents and permanent residents as a challenge accessing primary care services. ${ }^{\wedge}$ In addition, non-citizens and permanent residents had a lack of access to certain medico-social services.

"If really bedbound, it involves an ambulance. Ambulance is about S\$140 (US\$104) per round trip" (CCM 4)

"Um, recently I have a case um, of um, a Japanese boy with autism - he's not a Singaporean, so that, that complicates a bit of the funding issues for some of the services." (CCM 5)

"Cost. Ya, even with the CHAS (Community Health Assist Scheme)** card thing, there's still some cash involved." (CCM 6)

${ }^{\wedge}$ S Singapore citizens and permanent residents receive subsidies of $75 \%$ and $37.5 \%$ respectively for consultation fees at the polyclinics whereas non-residents do not receive any subsidy.

${ }^{*}$ CHAS enables all Singapore Citizens to receive subsidies for medical care at participating General Practitioner clinics.

\section{Inter-professional Factor}

\section{Inaccessibility to doctors directly}

CCMs spoke about their inability to contact doctors directly. Generic emails of polyclinics were inappropriate for urgent matters but there was no alternative platform for communication with the doctors.

"It's just that sometimes for urgent cases if we cannot find $y$ 'know, the particular person um, it makes it difficult for us, we don't know who else we can contact... Ya, who can link us up with the doctors. Because we know, we don't know which doctor will be seeing the patient, so sometimes we don't know who to talk to, ya.'(CCM 5) 
"... it's not only a system whereby we put in services, a system like, we can do communication. Like, we can leave a note there, if you think sensitive information, then we don't discuss in this platform, we probably, at least exchange number..." (CCM 11)

\section{Mismatched expectations}

CCMs described the difficulty and inconsistent criteria of obtaining a medical report from doctors. This was a common, recurring theme and barrier to the organization of social services for patients.

One CCM said with frustration "We can write the social report, but I just want someone to fill up the medical report!" (CCM 4)

"I mean have one example that the doctor also didn't want to fill up. That time I actually followed the patient there, but the doctor also didn't want to fill up... It really depends on your luck you know. Somesome doctors are okay, some are not." (CCM 5)

They explained this reticence as due to the doctors' time constraints and a concern about medical liability.

"Ya, so we need one doctor to write that. But I think there's a bit of liability issue, so-so some doctor is not, and not all polyclinic doctor will write the nursing home (medical) report." (CCM 4)

"And I don't know whether they you know, for first-time patient will they have time to do a full check-up and really to write the report out. I don't know the time, is it an issue? Ya." (CCM 5)

In addition, the majority of doctors seemed unaware of CCMs' crucial need for a medical report to move services within the community.

"So again, the limiting factor if they're not assessed and labeled, they cannot move, they cannot get a lot of support..." (CCM 4)

\section{Doctors' attitude}

CCMs spoke at length about the relationship between doctors and patients during a consultation that generated challenges to their role. They frequently described how doctors showed a lack of empathy to their patients and failed to recognize patients' preference.

"I do have some referrals from doctor who somehow thought that they've made the assessments. The problem is, when you see patients right, it's really the tip of the ice, of the iceberg." (CCM 1)

“... she couldn't see properly, and she doesn't has the strength to press the medicine out of the blister. So, like, she's not taking her medicines. So, these are the challenges that they face... the doctors, they prescribe medicine to um, um... the client, but then $y^{\prime}$ know, some might not have the means to follow the routine, or... (CCM 12) 
Relationship depended on trust and communication. Patients and caregivers needed time to build rapport. Often, patients were unable to share their concerns with the doctors and language was also a barrier to effective communication.

"I think in polyclinic it's very fast-paced. Each doctor doesn't have enough time with the patient... So sometimes it could be a language problem as well, because some of our elderly they speaks like, dialect..." (CCM 4)

"... I remember one doctor very well ... I think he's Malaysian, he can speak very good Malay. Really, make the... patient feel at ease. Because my patient, at that point of time, had some ah, depression symptoms. So I think that really helped. (CCM 9)

\section{Power dynamics}

Power dynamics emerged during the discussion with CCMs. They perceived that doctors were not interested and that their inputs about patients were not listened to.

"Er... If we see that they are don't really care, we'll just make it short. We're not going to bother you with too much... 30\% don't listen." (CCM 6)

"So sometime we want to go with them, but it's not every time the doctors will want to discuss cases with us. That's my experience." (CCM 11)

\section{Interaction with MSW}

As well as doctors, CCMs often tried to interact with MSWs but often this linking to primary care services also failed due to the unavailability of MSWs.

"Not that they are part-time, but, they only few days will be there. So, also changing social worker. So I don't have a regular person..." (CCM 11)

"Then secondly, they might not be based in the same polyclinic every day Ya, then, secondly, I'm not too sure about wha-what their roles are. Like, I'm not very sure." (CCM 12)

CCMs described their interactions with MSWs as being cordial, recognizing they shared similar goals, although MSWs were seen mostly dealing with financial issues.

"So far okay, but mostly it's just financial, they don't really deal with a lot of case management thing." (CCM 7)

"Whereas um... for the polyclinic, I think it's like, the patient is just, hoping to get the financial and then er, I give you whatever information that you need about my finances, whatever family support that I have? Er, beyond that er... they, they can't do anything much." (CCM 14)

\section{Collaborative culture}




\section{Continuity of care}

CCMs highlighted that lack of continuity of care was an issue they encountered, with a different doctor assessing the patients on each visit, making it difficult to establish professional rapport.

"... because polyclinic you don't know which doctor will be seeing that patient on that day, so it's hard for us to even $y^{\prime}$ know, find someone... Because right now, I don't know they, when they go back to see polyclinic, it's always different doctors, you see so..." (CCM 5)

"basically, the doctors they wouldn't know as well. Because er... you will be seeing different doctors every time you go, from the primary care." (CCM 14)

Fragmentation of care was also pointed out when multiple agencies were involved in the care of patients. These partners failed to work together in consolidating a harmonized patient care plan.

"This is something that we face, if there's too many... workers involved, um... it can be helpful, it can complicate the case." (CCM 13)

"er... nurses and us, er, sometimes - now that community nurse is coming in - it could be overlapping, and... could be confusing to... the patients, er, even among our own... [tsk] sector itself." (CCM 14)

At times, care integration programs were ad hoc and did not last.

"There's no, concrete, concrete structure in place, because there's no er, middle person to hold the... vessel in place. Because you set out a vessel right, when the head go off, everything dissolves already. That's the issue." (CCM 7)

\section{Care plan}

CCMs stated that there was rarely a single care plan that included all the stakeholders (e.g., multiple doctors caring for a patient with multiple conditions) in the care of complex patients. In addition, there was no ownership of the care plan and lack of precise handover information to the CCMs.

"... she was diagnosed with dementia. So, nothing done... Because if she's demented, then maybe need some care planning." (CCM 6)

"I think common issue really is about the... the care plan. Because it's like, somehow, if let's say, you have cases where, you have chronic disease then, um, multiple, then it's like, not taking good care, then, who are the healthcare... providers that we can work with" (CCM 11)

\section{Confidentiality}

\section{Balancing role as a patient advocate}


Patients tended to normalize their concerns when they were in a clinical consultation, leaving physicians unaware of the reality of the actual situation. CCMs described that it was difficult being a patient's advocate when patients did not share pertinent information with their healthcare providers. Respecting patient's autonomy and confidentiality limited CCMs ability to share with the clinicians the information they had about patients.

"... elderly tend to normalize, norm, normalize a lot of thing. When the doctor asks them, they will say, "Ho4 ("Good/I'm fine" in Chinese dialect). Then, when they at home right, they will tell us, "Aiya, here pain, there pain." (CCM 4)

"Sometimes I don't know whether me being there, does it help or actually, I actually do more harm, because the doctor will tend to just ask me more question instead of asking the patient directly." (CCM 5)

"There're doctors whereby you-you go in, then, they only want to talk to you. Like, they don't talk - the patient is there but they don't talk to the patient, they talk to me. Like, they will instruct me, like, they will ask like, how's the patient these few days?" (CCM 12)

\section{Limited access to medical information}

CCMs also spoke of their limited access to medical information due to the Personal Data Protection Act (PDPA), thus posing a challenge to obtain a proper diagnosis for medical and social care application.

"I mean, now in the system is that we cannot see anything. So sometimes you've to get, just get from the patient lo. That's not very, I mean to make it more effective it'd be

good that we can see what's being done, and what is the plan, ya. (CCM 3)

“... For nursing home daycare right, you want to go a dementia one, you need a diagnosis. If you have no diagnosis right, you cannot go to, dementia daycare; you cannot go to dementia nursing home, which is very important for us as well." (CCM 4)

\section{Organizational structure}

\section{Different services and workflows}

CCMs elaborated on the confusion they experienced navigating different workflows in each polyclinic cluster. Service variation and availability offered were also a challenge with primary care services.

"Polyclinic Cluster $X$ right, the, the nursing home application can only be done on a Saturday only. Because they allocated doctor to do this on a Saturday... Polyclinic Cluster $Y$ is, I think, generally all doctor can do, ya. Mm, but we want to highlight to social worker to secure, I mean, to make sure that it really can be done." (CCM 4)

"Maybe more of the I think process is one of it, because different polyclinics have different, different clusters have different processes, so we get confused at times as well." (CCM 5) 
Different workflows also resulted in confusion of staffs' job role and contact person, as illustrated below.

"Okay, from what I understand right, um, actually financial counsellor is a new concept right, last time don't have." (CCM 4)

"They're, if I'm not wrong they are nurses, if not nurses, nursing coordinator... Is it a care coordinator? I forgot what is their role. There's so many, I also don't know who are they, ya." (CCM 5)

\section{Inflexibility of workflows}

CCMs shared that frequently, the systematic workflow resulted in inflexibility to access personalized care. This was illustrated in making appointments and accessing specific services.

"So the patient has to make a special trip just to apply for maybe a day care service. But maybe on Tuesday he's already went, gone down to s, the polyclinic to $y$ 'know, follow up on chronic conditions... it makes it tough on the patient, especially if they have problems even going down to see the doctor." (CCM 5)

"... because polyclinic I need to go for blood test, I need to see doctor, I need to, one week later, a lot of things, then social worker need to see you on another day, blah blah blah, so that one a bit messy, ya." (CCM 6)

\section{Discussion}

The challenges faced by CCMs in collaborating with primary care services stem from various factors, including self-identity, patient characteristics, inter-professional issues, collaborative culture, confidentiality and organizational structure. Nonetheless, all interviewees were committed to the concept of collaboration despite the multiple challenges. CCMs want to be understood and to be involved in the care of patients. The satisfaction of journeying with patients outweighed the lack of their current professional recognition. CCMs emphasized the need for their continuing involvement and a shared care plan for complex patient that involves social and medical input.

Unfortunately, CCMs perceived that they were of little value in the primary care arena reflected by the physicians' attitudes and communication with them. They perceived physicians retaining a very dominant role in the planning of healthcare, whereby CCMs provide information as and when requested, but not as collaborative partners in the on going care of patients in the community. Though both professionals focused on patients at the center of their care, much has to be worked on together in aligning their paradigm. More can be done to focus on collegial interaction skills on top of clinical encounter skills.

This study's findings resonate with previous studies of Netting \& Williams [9] and Keefe, B. [19] on the challenges of case managers' struggles with professional relationships, roles and identity. This reinforced that the role and self-identity of CCMs issues are generic and not institution specific. In addition, the 
themes on confidentiality and organizational structure were in line with the discussion of Gabrielova \& Veleminsky [18]. Confidentially and organizational issues transcend all aspects of medical care and CCMs are not spared either.

Albeit these similarities, this study contributes a new perspective of CCMs' challenges with primary care services, notably the inaccessibility to doctors, mismatched expectations and their interaction with the MSWs. CCMs also spoke at length about the challenges with regard to patients of intellectual and physical disability seeking primary care services. Collaborative culture that was discussed in AmbroseMiller \& Ashcroft [22] study differs to this study. In this study, CCMs were concerned of continuity of care and care plan of patients. Paradoxically, the easy accessibility to primary care does not necessarily translate to better continuity of care with a regular doctor due to time, manpower and financial constraints. Inter-professional communication was a major theme in studies of Ambrose-Miller \& Ashcroft [22] and Abramson \& Mizrahi [23]; however, in this study, CCMs were concerned with the communication between physician and patient instead. Inter-professional communication did arise, albeit in a more muted respond and was explored under the sub-themes of power dynamics among professionals.

There is a need for community case management to play a greater role in Singapore's changing health care landscape of 'Beyond Hospital to Community', but issues of confidentiality, collaborative culture and inter-professional factor currently deter the integration of primary care and social care. Formal recognition of CCMs through a statutory board to govern registered CCMs would be a step in the right direction to alleviate many of these obstacles arising from lack of recognition.

In addition, as the health care system moves toward greater emphasis on ambulatory settings, workflows and medical information technology need to be revised to cater for non-medical professionals who are directly involved with patient care. Gone are the days when physicians worked alone without interprofessional collaboration. Complex patients need a common care plan with constant input from CCMs; thus an overarching medical record comprising of social, financial and medical elements is a sine qua non for successful care integration.

CCMs and primary care services need to develop meaningful and sustained relationships instead of episodic collaborations. Mismatched expectations between CCMs and primary care physicians do arise accordingly as in this study; nevertheless, success in collaborative practice requires stakeholders to understand these barriers and strategize to minimize them. Emphasizing uniqueness and differences may separate the professionals at a time when it is more imperative to focus on the patient.

A change from current practice through continuous dialogue and appreciation of each other's professional role will take time. Parallel change needs to take place during the early formation of CCMs and medical professionals. Thus, educators need to be innovative with the current undergraduate curriculum. The school of medicine and the school of social work should collaborate to ensure students understand each other's domain and challenges through attachments or joint projects. Consequently, both will learn to speak each other's language for the benefit of patients. 


\section{Strengths \& Limitations}

This is a novel study for Singapore with regard to CCMs and primary care services. The reliability of the data was enhanced by the transparent process of analysis, the involvement of two independent researchers who read and compared ideas on the transcripts.

Nevertheless, there are limitations of this study that need highlighting. The individual interviews were carried out by a primary care physician, which may influence CCMs' responses about professional communication with doctors. It would have been ideal to have a CCM in the interview team to reassure the interviewee and address any power imbalance. Our prior experience and knowledge of working with CCMs may have inadvertently influenced our lenses of interpretation that may differ if it was interpreted by CCMs themselves.

Future studies are now needed to evaluate medical professionals' perspective on CCMs and their impact on primary care. This dyad will ensure patient-centered outcomes to be the focus in community care.

\section{Conclusion}

This study has identified several challenges that CCMs faced with primary care services in Singapore. Understanding these challenges is key to refining the current models of comprehensive community care between medical and non-medical profession to address bio-psychosocial aspect of care. Dissemination of the findings will play an important stimulus in ensuring continuing engagement and building a stronger collaboration to meet the needs of complex patients.

\section{Declarations}

\section{Ethics Approval \& Consent to Participate}

Ethics approval for this exploratory qualitative descriptive study was obtained from the National Healthcare Group Domain Specific Review Board (NHG DSRB) (Ref: 2018/00801) and methods were performed in accordance with the relevant guidelines and regulations laid by NHG DSRB.

\section{Consent for Publication}

Not applicable, manuscript does not contain data from any individual person.

\section{Availability of Data \& Materials}

The datasets used and/or analysed during the current study are available from the corresponding author on reasonable request. 


\section{Competing Interest}

The authors declare that they have no competing interests.

\section{Funding}

This research was supported by the Singapore Ministry of Health's National Medical Research Council under the Centre Grant Programme (Ref No: CGAug16C019).

\section{Authors' Contributions}

GTS Yeo and PK performed the interviews, data collection and data analysis.

ES Lee and HE Smith verified the analytical methods and supervised the findings of this work. GTS Yeo wrote the main manuscript text. All authors reviewed the manuscript.

\section{Acknowledgements}

This research was supported by administrative colleagues of Clinical Research Unit, National Healthcare Group Polyclinics.

\section{References}

1. Valderas, J.M., et al., Defining comorbidity: implications for understanding health and health services. Ann Fam Med, 2009. 7(4): p. 357 - 63. PMID: 19597174

2. Zulman, D.M., et al., Quality of care for patients with multiple chronic conditions: the role of comorbidity interrelatedness. J Gen Intern Med, 2014. 29(3): p. 529 - 37. PMID: 24081443

3. Grembowski, D., et al., A conceptual model of the role of complexity in the care of patients with multiple chronic conditions. Med Care, 2014. 52 Suppl 3: p. S7-S14. PMID: 24561762

4. Community Case Management Service (CCMS), the Agency for Integrated Care (AIC). (n.d). Retrived from http://feiyue-wpengine.netdna-ssl.com/wp-content/uploads/2019/07/CCMS-Referral-Formv6.pdf

5. Primary Care Survey 2014, Ministry of Health, Singapore. (n.d). Retrived from https://www.moh.gov.sg/docs/librariesprovider5/ resources-statistics/reports/moh-primary-caresurvey-2014-report.pdf

6. Agency for Integrated Care, Community Case Management Service (CCMS) Case Managers Go the Distance. (2019) Retrived from https://aic-mosaic.sg/2019/01/17/community-case-managementsystem-ccms-case-managers-go-the-distance/ 
7. Case Management Society of Singapore, Definition Of Case Management. (2019). Retrieved from https://www.casemanagement.sg/About_Us/Definition_of_Case_Management/

8. Kodner, D.L., Whole-system approaches to health and social care partnerships for the frail elderly: an exploration of North American models and lessons. Health Soc Care Community, 2006. 14(5): p. 384 - 90. PMID: 16918830

9. Netting, F.E. and F.G. Williams, Case manager-physician collaboration: implications for professional identity, roles, and relationships. Health Soc Work, 1996. 21(3): p. 216 - 24. PMID: 8854126

10. Geron, S.M. (2000). Care management in the United States. In M. White \& R. Applebaum (eds.), Key issues in case management around the globe. San Francisco, CA: American Society on Aging. ISBN: 0964038730

11. Sommers, L.S., et al., Physician, nurse, and social worker collaboration in primary care for chronically ill seniors. Arch Intern Med, 2000. 160(12): p. 1825-33. PMID: 10871977

12. Claiborne, N., Effectiveness of a care coordination model for stroke survivors: a randomized study. Health Soc Work, 2006. 31(2): p. 87-96. PMID: 16776026

13. Nikolaus, T., et al., A randomized trial of comprehensive geriatric assessment and home intervention in the care of hospitalized patients. Age Ageing, 1999. 28(6): p. 543 - 50. PMID: 10604506

14. Williams, M.E., et al., How does the team approach to outpatient geriatric evaluation compare with traditional care: a report of a randomized controlled trial. J Am Geriatr Soc, 1987. 35(12): p. 1071-8. PMID: 3119693

15. Rizzo, V.M. and J.M. Rowe, Studies of the cost-effectiveness of social work services in aging: A Review of the Literature. Research on Social Work Practice, 2016. 16(1): p. 67-73. doi: $10.1177 / 1049731514563578$

16. Tajfel, H., \& Turner, J.C. (1986). The social identity theory of inter-group behavior. In S. Worchel \& L.W. Austin (eds.), Psychology of intergroup relations (pp. 7-19). Chigago: Nelson-Hall. ISBN: 0818502789

17. Abramson, J.S. and T. Mizrahi, Understanding collaboration between social workers and physicians: application of a typology. Soc Work Health Care, 2003. 37(2): p. 71-100. PMID: 12959487

18. Gabrielova, J. and M. Veleminsky, Sr., Interdisciplinary collaboration between medical and nonmedical professions in health and social care. Neuro Endocrinol Lett, 2014. 35 Suppl 1: p. 59-66. PMID 25433356

19. Keefe, B., S.M. Geron, and S. Enguidanos, Integrating social workers into primary care: physician and nurse perceptions of roles, benefits, and challenges. Soc Work Health Care, 2009. 48(6): p. $579-96$. PMID: 19860293

20. Ministry of Health Singapore, Integration of Health and Social Services to Support Seniors (2018). Retrieved from https://www.moh.gov.sg/news-highlights/details/integration-of-health-and-socialservices-to-support-seniors.

21. Topic Guide, Community Case Managers' (CCMs) perspectives' collaborating with primary care services when managing complex patients in the community: a qualitative study. (as attached in 
supplementary file)

22. Ambrose-Miller, W. and R. Ashcroft, Challenges faced by social workers as members of interprofessional collaborative health care teams. Health Soc Work, 2016. 41(2): p. 101-9. PMID: 27263200

23. Abramson, J.S. and T. Mizrahi, When social workers and physicians collaborate: positive and negative interdisciplinary experiences. Soc Work 41, 1996. 37(2): p. 270-283. PMID: 8936083

\section{Supplementary Files}

This is a list of supplementary files associated with this preprint. Click to download.

- TopicGuideV3d1.docx 